\title{
Publisher Correction to: Signomial and polynomial optimization via relative entropy and partial dualization
}

\author{
Riley Murray $^{1} \cdot$ Venkat Chandrasekaran $^{1} \cdot$ Adam Wierman $^{1}$ \\ Published online: 9 February 2021 \\ ๑) Springer-Verlag GmbH Germany, part of Springer Nature and Mathematical Optimization Society 2021
}

\section{Publisher Correction to: Mathematical Programming Computation https://doi.org/10.1007/s12532-020-00193-4}

In the original publication of the article, the reference list in the pdf version has been published with an error. The correct reference list is given in this correction.

The original article has been updated.

\section{References}

1. Rountree, D.H., Rigler, A.K.: A penalty treatment of equality constraints in generalized geometric programming. J. Optim. Theory Appl. 38(2), 169-178 (1982). issn: 1573-2878

2. Kirschen, P.G., et al.: Application of signomial programming to aircraft design. J. Aircr. 55(3), 965987 (2018)

3. Jabr, R.A.: Inductor design using signomial programming. COM-PEL Int. J. Comput. Math. Electr. Electron. Eng. 26(2), 461-475 (2007)

4. Chiang, M.: Nonconvex optimization for communication networks. In: Honor of Gilbert Strang, Advances in Applied Mathematics and Global Optimization, Springer US, Boston, pp. 137-196. ISBN: 978-0-387-75714-8 (2009)

5. Shen, P., Zhang, K.: Global optimization of signomial geometric programming using linear relaxation. Appl. Math. Comput. 150(1), 99-114 (2004)

6. Wang, Y., Liang, Z.: A deterministic global optimization algorithm for generalized geometric programming. Appl. Math. Comput. 168(1), 722-737 (2005). issn: 0096-3003

7. Shen, P., Jiao, H.: A new rectangle branch-and-pruning approach for generalized geometric programming. Appl. Math. Comput. 183(2), 1027-1038 (2006)

The original article can be found online at https://doi.org/10.1007/s12532-020-00193-4.

\author{
Riley Murray \\ rmurray@caltech.edu \\ Venkat Chandrasekaran \\ venkatc@caltech.edu \\ Adam Wierman \\ adamw@caltech.edu \\ 1 California Institute of Technology, Pasadena, CA 91125, USA
}


8. Shao-Jian, Q., Zhang, K.-C., Ji, Y.: A new global optimization algorithm for signomial geometric programming via Lagrangian relaxation. Appl. Math. Comput. 184(2), 886-894 (2007)

9. Shen, P., Ma, Y., Chen, Y.: A robust algorithm for generalized geometric programming. J. Global Optim. 41(4), 593-612 (2008). issn: 1573-2916

10. Hou, X., Shen, P., Chen, Y.: A global optimization algorithm for signomial geometric programming problem. Abstract Appl. Anal. 2014, 1-12 (2014)

11. Gongxian, X.: Global optimization of signomial geometric programming problems. Eur. J. Oper. Res. 233(3), 500-510 (2014)

12. Shor, N.Z.: Class of global minimum bounds of polynomial functions. Cybernetics 23(6), 731-734 (1987)

13. Parrilo, P.: Structured semidefinite programs and semialgebraic geometry methods in robustness and optimization. PhD thesis, California Institute of Technology (2000)

14. Lasserre, J.B.: Global optimization with polynomials and the problem of moments. SIAM J. Optim. 11(3), 796-817 (2001)

15. Chandrasekaran, V., Shah, P.: Relative entropy relaxations for signomial optimization. SIAM J. Optim. 26(2), 1147-1173 (2016)

16. Murray, R., Chandrasekaran, V., Wierman, A.: Newton polytopes and relative entropy optimization (2018). arXiv: 1810.01614

17. Iliman, S., de Wolff, T.: Amoebas, nonnegative polynomials and sums of squares supported on circuits. Res. Math. Sci. 3, 9 (2016)

18. Murty, K.G., Kabadi, S.N.: Some NP-complete problems in quadratic and nonlinear programming. Math. Program. 39(2), 117-129 (1987). issn: 1436-4646

19. MOSEK ApS. MOSEK 9.0.70(beta) (2019)

20. Reznick, B.: Forms derived from the arithmetic-geometric inequality. Math. Ann. 283(3), 431-464 (1989)

21. Pébay, P.P., Rojas, J.M., Thompson, D.C.: Optimization and NP R-completeness of certain fewnomials. In: Proceedings of the 2009 Conference on Symbolic Numeric Computation, ACM Press (2009)

22. Pantea, C., Koeppl, H., Craciun, G.: Global injectivity and multiple equilibria in uni- and bi-molecular reaction networks. Discrete Contin. Dyn. Syst. Ser. B 17(6), 2153-2170 (2012)

23. August, E., Craciun, G., Koeppl, H.: Finding invariant sets for biological systems using monomial domination. In: 2012 IEEE 51st IEEE Conference on Decision and Control (CDC), IEEE (2012)

24. Wang, J.: Nonnegative polynomials and circuit polynomials (2018). arXiv:1804.09455

25. Katthän, L., Naumann, H., Theobald, T.: A unified framework of SAGE and SONC polynomials and its duality theory (2019). arXiv:1903.08966

26. Seidler, H., de Wolff, T.: An experimental comparison of SONC and SOS certificates for unconstrained optimization (2018), arXiv:1808.08431

27. Seidler, H., de Wolff, T.: POEM: effective methods in polynomial optimization, version 0.2.1.0(a) (2019). http://www.iaa.tu-bs.de/AppliedAlgebra/POEM/index.html

28. Henrion, D., Lasserre, J.-B., Löfberg, J.: GloptiPoly 3: moments, optimization and semidefinite programming. Optim. Methods Softw. 24(4-5), 761-779 (2009)

29. Papachristodoulou, A., et al.: SOSTOOLS version 3.00 sum of squares optimization toolbox for MATLAB (2013). arXiv:1310.4716

30. Powers, V., Reznick, B.: Polynomials that are positive on an interval. Trans. Am. Math. Soc. 352(10), 4677-4692 (2000)

31. Laurent, M.: Sums of squares, moment matrices and optimization over polynomials. In: Putinar, M., Sullivant, S. (eds.) Emerging Applications of Algebraic Geometry, pp. 157-270. Springer, New York (2009). isbn: 978-0-387-09686-5

32. Borwein, J., Lewis, A.: Convex Analysis and Nonlinear Optimization. Springer, New York (2006)

33. Lasserre, J.B.: An Introduction to Polynomial and Semi-algebraic OptimizationCambridge Texts in Applied Mathematics. Cambridge University Press, Cambridge (2015)

34. Powell, M.J.D.: A direct search optimization method that models the objective and constraint functions by linear interpolation. In: Advances in Optimization and Numerical Analysis, Springer, Dordrecht, pp. 51-67. ISBN: 978-94-015-8330-5 (1994)

35. Yan, J.: Signomial programs with equality constraints: numerical solution and applications. $\mathrm{PhD}$ thesis, University of British Columbia (1976)

36. Agrawal, A., Diamond, S., Boyd, S.: Disciplined geometric programming. Optim. Lett. 13(5), 961976 (2019) 
37. Bard, G.V.: Some basic facts about linear algebra over GF(2). In: Algebraic Cryptanalysis, Springer, Berlin, pp. 81-88 (2009)

38. Verschelde, J.: Algorithm 795: PHCpack-a general-purpose solver for polynomial systems by homotopy continuation. ACM Trans. Math. Softw. 25(2), 251-276 (1999). issn: 0098-3500

39. Ray, S., Nataraj, P.S.V.: An efficient algorithm for range computation of polynomials using the Bernstein form. J. Global Optim. 45(3), 403-426 (2008)

40. Lasserre, J.B., Toh, K.-C., Yang, S.: A bounded degree SOS hierarchy for polynomial optimization. EURO J. Comput. Optim. 5(1), 87-117 (2017). issn: 2192- 4414

41. Weisser, T., Lasserre, J.B., Toh, K.-C.: Sparse-BSOS: a bounded degree SOS hierarchy for large scale polynomial optimization with sparsity. Math. Program. Comput. 10(1), 1-32 (2018). issn: $1867-2957$

42. Murray, R.: Sageopt 0.5.3 (2020). https://doi.org/10.5281/ZENODO.4017991

43. Domahidi, A., Chu, E., Boyd, S.: ECOS: an SOCP solver for embedded systems. In: European Control Conference (ECC), pp. 3071-3076 (2013)

44. Serrano, S.A.: Algorithms for unsymmetric cone optimization and an implementation for problems with the exponential cone. PhD Thesis, Stanford University, Palo Alto, CA (2015)

45. Burnell, E., Damen, N.B., Hoburg, W.: GPkit: a human-centered approach to convex optimization in engineering design. In: Proceedings of the 2020 CHI Conference on Human Factors in Computing Systems (2020)

46. Rijckaert, M.J., Martens, X.M.: Comparison of generalized geometric programming algorithms. J. Optim. Theory Appl. 26(2), 205-242 (1978). issn: 1573-2878

47. Surjanovic, S., Bingham, D.: Virtual library of simulation experiments: test functions and datasets. Retrieved April 18 from http://www.sfu.ca/ ssurjano (2019)

48. Ahmadi, A.A., Majumdar, A.: DSOS and SDSOS optimization: more tractable alternatives to sum of squares and semidefinite optimization. SIAM J. Appl. Algebra Geom. 3(2), 193-230 (2019)

49. Vandenberghe, L.: The CVXOPT linear and quadratic cone program solvers (2010). http://www. seas.ucla.edu/ vandenbe/publications/coneprog.pdf

50. Forsgård, J., de Wolff, T.: The algebraic boundary of the sonc cone (2019). arXiv:1905.04776

Publisher's Note Springer Nature remains neutral with regard to jurisdictional claims in published maps and institutional affiliations. 